Popper: Ueber die quantitative Bestimmung von Niederschlägen etc. 157

Ueber die quantitative Bestimmung von Niederschlägen ohne Auswaschen und Trocknen derselben.

$$
\text { Von }
$$

\title{
Richard Popper.
}

Es ist bekannt, dass, wenn man einen aus einer Lösung gefällten Niederschlag durch Auswaschen von dieser Lösung befreit, ihn hierauf vom Filter in ein Pyknometer "spült, dieses mit Wasser füllt und wägt, das Gewicht des Niederschlags berechnet werden kann.

Hierzu ist es nöthig, dass sein spec. Gewicht bekannt sei und man wisse, wieviel das mit Wasser gefüllte Pyknometer allein wiegt.

Dieses Verfahren gewährt jedoch, wie jeder bald erkennt, keine grossen Vortheile, indem dasselbe vor dem gewöhnlichen nur den Vorzug hat, dass man den auf dem Filter befindlichen Niederschlag nicht erst zu trocknen braucht. Uebrigens ist das spec. Gewicht eines Niederschlags nicht immer ganz constant, wenn auch die hierdurch entstehenden geringen Ungenauigkeiten in den meisten Fällen ganz gut vernachlässigt werden könnten.

Vor Kurzem hatte ich nun das Glück, ein Verfahren zu finden, mit Hülfe dessen ausser dem Trocknen auch das Auswaschen leicht umgangen werden kann.

Wenn man nämlich den Niederschlag mit derselben Flüssigkeit ins Pyknometer spült, in der er ausgefällt ist, so lässt sich sein Gewicht sehr gut berechnen, sobald man nur, ausser dem durch einen Fundamentalversuch festgestellten spec. Gewicht desselben, auch das specifische Gewicht der Lösung kennt, was sich ja durch eine einzige Wägung finden lässt.

Es sei das durch einen Fundamentalversuch gefundene spec. Gewicht des betreffenden Niederschlags $\mathbf{S}$, das spec. Gewicht der Lösung, in welcher sich der Niederschlag nach dem Ausfällen befindet, $s$, - ferner das Gewicht des Pyknometers sammt Lösung und Niederschlag G, das Gewicht Ces Pyknometers mit der Lösung allein gefüllt $g$, und endlich das $z u$ suchende Gewicht des Niederschlags $x$, so ergibt sich folgende Gleichung

$$
\mathrm{G}=\mathrm{g}+\mathrm{x}-\frac{\mathrm{x}}{\mathrm{S}} \mathrm{s} \text {. }
$$

Denn die Grösse $\frac{\mathrm{x}}{\mathrm{S}}$ stellt ja das Volumen der vom Niederschlag ver- 
drängten Lösung, folglich $\frac{\mathrm{x}}{\mathrm{S}} \mathrm{s}$ ihr Gewicht dar, welches natürlich in $\mathrm{Ab}$ zug gebracht werden muss.

Aus der obigen Gleichung ergibt sich

$$
\mathrm{x}-\frac{\mathrm{x}}{\mathrm{S}} \mathrm{s}=\mathrm{G}-\mathrm{g}
$$

und hieraus durch Umformung

$$
\begin{aligned}
& \mathrm{x} S \sim \mathrm{xs}=\mathrm{S}(\mathrm{G}-\mathrm{g}), \\
& \text { folglich } \mathrm{x}=\frac{\mathrm{S}}{\mathrm{S}-\mathrm{s}}(\mathrm{G}-\mathrm{g}) .
\end{aligned}
$$

Die hierin vorkommenden Werthe $G$ und $g$ ergeben sich direkt aus der Wägung, $\mathrm{S}$ ist bekannt und $\mathrm{s}$ ergibt sich, wenn man von $\mathrm{g}$ die Tara des Pyknometers subtrahirt und die erhaltene Differenz durch das bekannte Volumen desselben dividirt. Zur wirklichen Ausführung solcher Versuche wäblte ich den Thonerdeniederschlag, weil bei diesem ein Ersparen des Auswaschens doch gewiss wünschenswerth sein würde, indem er sich bekanntlich sehr schlecht von der anhängenden Lösung befreien lässt, und weil ausserdem für diesen Körper, obgleich er ziemlich häufig ist, eine brauchbare, maassanalytische Methode, wie bei Eisen, Kalk u. a. nicht existirt.

Es war natürlich, ehe ich mit diesen Versuchen begann, ein von fremden Bestandtheilen freies Thonerdesalz nöthig.

Da dies nicht vorhanden war, so musste es durch mehrmaliges Umkrystallisiren hergestellt werden. Ich wählte hierzu den Kalialaun, weil dieser am leichtesten auskrystallisirt und sich also auch am leichtesten rein herstellen lässt. Dieses Umkrystallisiren wurde fortgesetzt, bis sich die Alaunkrystalle bei einer Prüfung auf Eisen frei davon zeigten.

Um nun vor Allem das spec. Gewicht des Thonerdeniederschlages festzustellen und ausserdem zu sehen, ob dasselbe nicht etwa in verschie. denen Fällen zu sehr differire, stellte ich mir grössere Mengen Thonerdeniederschlag her, die ich aus 2 Lösungen von sehr verschiedener Concentration fällte.

Diese Niederschläge wurden durch öfter wiederholte Dekantation so lange ausgewaschen, bis das abfliessende Wasser auf Zusatz von Chlorbaryum sich nicht mehr trübte.

Ich dampfte nun das noch im Becherglas zurückgebliebene ungefähr zur Hälfte ein und füllte, nachdem es ausgekühlt war, mein Pyknometer damit. Jeder der beiden Niederschläge, also sowohl der aus der ver- 
dünnten, als auch der aus der concentrirten Lösung gefällte, reichten für 2 Pyknometerfüllungen, also beide zusammen für 4 Versuche hin.

Ich nahm nun die 4 Wägungen vor, nachdem jedesmal vorher die Temperatur gemessen und das gefundene Gewicht auf die Temperatur von $17,5^{0}$ reducirt worden war.

Nach jeder Wägung spülte ich das im Pyknometer befindliche auf ein Filter und fand in dieser Weise die Menge der angewandten Thonerde.

Aus dem Gewicht eines Körpers in und ansserhalb des Wassers lässt sich aber bekanntlich sein spec. Gewicht leicht berechnen, wie folgt:

Das Gewicht des mit dem Niederschlage und Wasser gefüllten Pyknometers wäre offenbar gleich

$$
\text { Pykn. + Wasser + N }-\frac{\mathrm{N}}{\mathrm{S}}
$$

wenn $\mathrm{N}$ die Menge des Niederschlags und $\mathrm{S}$ das noch unbekannte spec. Gewicht desselben bedeutet.

Subtrahirt man das Gewicht des mit Wasser gefüllten Pyknometers von obigem Werthe, so würde offenbar übrig bleiben

$$
\mathrm{N}-\frac{\mathrm{N}}{\mathrm{S}} \text {. }
$$

Nehmen, wir an, es sei dieser Werth gleich $M$ gefunden worden, so entstände also die Gleichung

$$
\mathrm{N} \rightarrow \frac{\mathrm{N}}{\mathrm{S}}=\mathrm{M} \text {. }
$$

Da $\operatorname{man} \mathrm{N}$ durch die Gewichtsanalyse in der vorhin erwähnten Weise finden kann, so enthält die Gleichung nur eine Unbekannte $\mathbf{S}$, welche sich leicht ausdrücken lässt. Durch Umformung erhält man nämlich aus obiger Gleichung

$$
\mathrm{N} \mathrm{S}-\mathrm{N}=\mathrm{M} \mathrm{S}
$$

und hieraus

$$
(\mathrm{N}-\mathrm{M}) \mathrm{S}=\mathrm{N}
$$

also

$$
\mathrm{S}=\frac{\mathrm{N}}{\mathrm{N}-\mathrm{M}}
$$

Es ergaben sich nun bei den Versuchen folgende Resultate:

I. Thonerde aus der verdünnten Alaunlösung

a. Pykn. + Wasser $+\mathrm{Al} \theta_{3} \mathrm{H}_{3}-\frac{\mathrm{Al} \theta_{3} \mathrm{H}_{3}}{\mathrm{~S}}=50,5784$ (Temp. 22,50) Pykn. + Wasser $=50,1180$ (Temp. 21,80). 
Beides auf die Temperatur von $17,5^{0}$ reducirt, gab, da ich gefunden hatte, dass für mein mit Wasser gefülltes Pyknometer einer Temperaturzunahme von $1^{0} \mathrm{C}$., eine Gewichtsabnahme von 8 Milligramm entspreche (der Ausdehnungscoefficient des Niederschlags, welcher an Volumen kaum 1/100 der Flüssigkeit einnahm, konnte natürlich vollständig vernachlässigt werden):

$$
\begin{aligned}
\mathrm{P}+\mathrm{W}+\mathrm{Al} \theta_{3} \mathrm{H}_{3}-\frac{\mathrm{Al} \theta_{3} \mathrm{H}_{3}}{\mathrm{~S}}=50,5784-5.0,008=50,5384 \mathrm{Grm} . \\
\mathrm{P}+\mathrm{W}=50,118-4,3.0,008=50,0846 \mathrm{Grm} . \\
\mathrm{Al} \theta_{3} \mathrm{H}_{3}-\frac{\mathrm{All} \theta_{3} \mathrm{H}_{3}}{\mathrm{~S}}=\mathbf{0 , 4 5 3 8 \mathrm { Grm } .}
\end{aligned}
$$

Ferner ergab sich hierbei

$$
\begin{aligned}
\text { Tiegel }+\mathrm{Asche}+\mathrm{Al}_{2} \theta_{3} & =24,0649 . \\
\text { Tiegel }+\mathrm{Asche} & =23,5225 \\
\mathrm{Al}_{2} \theta_{3} & =0,5424 \\
\mathrm{Al} \theta_{3} \mathrm{H}_{3} & =0,8273 .
\end{aligned}
$$

also

Nach der entwickelten Formèl würde man also haben

$$
\mathrm{S}=\frac{0,8273}{0,8273-0,4538}
$$

Hieraus ergibt sich

$$
\mathrm{S}=2,215 \text {. }
$$

b. $\mathrm{P}+\mathrm{W}+\mathrm{Al} \Theta_{3} \mathrm{H}_{3}-\frac{\mathrm{Al} \Theta_{3} \mathrm{H}_{3}}{\mathrm{~S}}=50,505$ (Temp. 21,00 C.)

Dies wäre bei $17,5^{0}$

$$
\begin{array}{rl}
\mathrm{P}+\mathrm{W} & \mathrm{Al} \theta_{3} \mathrm{H}_{3}-\frac{\mathrm{Al} \theta_{3} \mathrm{H}_{3}}{\mathrm{~S}}=50,595-3,5 \cdot 0,008=50,5670 \\
& \mathrm{Al} \theta_{3} \mathrm{H}_{3}-\frac{\mathrm{Al} \theta_{3} \mathrm{H}_{3}}{\mathrm{~S}}=0,4824 .
\end{array}
$$

Ausserdem ergab sich

$$
\begin{array}{r}
\text { Tiegel }+ \text { Asche }+\mathrm{Al}_{2} \theta_{3}=24,0980 \\
\text { Tiegel }+\mathrm{Asche}=23,5225 \\
\mathrm{Al}_{2} \theta_{3}=0,5755
\end{array}
$$

und hieraus

$$
\mathrm{A}-1 \Theta_{3} \mathrm{H}_{3}=0,8778
$$

also

$$
\begin{aligned}
& \mathrm{S}=\frac{0,8778}{0,8778-0,4824} \\
& \mathrm{~S}=2,220 .
\end{aligned}
$$


II. Thonerde aus der concentrirten Alaunlösung.

a. $\mathrm{P}+\mathrm{W}+\mathrm{Al} \theta_{3} \mathrm{H}_{3}-\frac{\mathrm{Al} \Theta_{3} \mathrm{H}_{3}}{\mathrm{~S}}=50,7215$ (reducirt anf $17,5^{\circ} \mathrm{C}$ )

$$
\begin{aligned}
\mathrm{P}+\mathrm{W} & =50,0846 \\
\mathrm{Al} \mathrm{\theta}_{3}, \mathrm{H}_{3}-\frac{\mathrm{All} \Theta_{3} \mathrm{H}_{3}}{\mathrm{~S}} & =0,6369 .
\end{aligned}
$$

Ausserdem fand sich

$$
\begin{aligned}
\mathrm{T}+\mathrm{A}+\mathrm{Al}_{2} \Theta_{3} & =24,2865 \\
\mathrm{~T}+\mathrm{A} & =23,5225 \\
\mathrm{Al}_{2} \Theta_{3} & =0,7640 \\
\mathrm{Al} \theta_{3} \mathrm{H}_{3} & =1,1654 . \\
\mathrm{S} & =\frac{1,1654}{1,1654-0,6369} \\
\mathrm{~S} & =2,205 .
\end{aligned}
$$

b. $P+W+A l \theta_{3} H_{3}-\frac{A l \theta_{3} H_{3}}{S}=50,6043$ (reducirt auf $17,5^{0}$ )

$$
\begin{aligned}
\mathrm{P}+\mathrm{W} & =50,0846 \\
\text { A.l } \theta_{3} \mathrm{H}_{3}-\frac{\mathrm{All} \theta_{3} \mathrm{H}_{3}}{\mathrm{~S}} & =0,5197 .
\end{aligned}
$$

Ferner ergab sich

$$
\begin{aligned}
\mathrm{T}+\mathrm{A}+\mathrm{Al}_{2} \theta_{3} & =24,1526 \\
\mathrm{~T}+\mathrm{A} & =23,5225 . \\
\mathrm{Al}_{2} \theta_{3} & =0,6301
\end{aligned}
$$

und hieraus

$$
\begin{aligned}
& \text { Al } \Theta_{3} \mathrm{H}_{3}=0,9474 . \\
& \mathrm{S}=\frac{0,9474}{0,9474-0,5197} \\
& \mathrm{~S}=2,215 .
\end{aligned}
$$

Die spec. Gewichte der aus der verdünnten Lösung gefällten Thonerde waren also

$$
2,215 \text { und } 2,220
$$

und die spec. Gewichte der aus der concentrirten Lösung gefällten

$$
2,205 \text { und } 2,215 \text {. }
$$

Alle diese Zahlen stimmen wenigstens soweit überein, dass jedenfalls für technische Analysen vollkommen genügende Resultate damit erzielt werden könnten.

Die kleinen hier vorliegenden Ungenauigkeiten kommen auch wahrscheinlich nicht von einer Verschiedenheit des spec. Gewichts der Thon- 
erde, wenn sie aus verschieden concentrirten Lösungen gefällt wird, sondern rühren jedeufalls von den unvermeidlichen Fehlerquellen der mir zu Gebote stehenden Instrumente her, denn es zeigen sich ja oben zwischen den spec. Gewichten der ans einer Lösung gefällten Thonerde dieselben kleinen Differenzen, wie zwischen den spec. Gewichten der aus verschiedenen Lösungen erhaltenen.

Das mittlere spec. Gewicht des Thonerdeniederschlages wäre nach den obigen Zahlen

$$
2,214 \text {. }
$$

Nachdem nun in dieser Weise das spec. Gewicht des Thonerdeniederschlages festgestellt war, konnte zur wirklichen Ausführung der Analysen geschritten werden.

Ich wog deshalb $40 \mathrm{Grm}$. Alaun ab, löste sie in Wasser zu einem Liter und nahm hiervon mit einer Pipette 2 mal $50 \mathrm{CC}$. heraus, fällte nach Zusatz von etwas Salmiak die Thonerde in der bekannten Weise mit Ammoniak und erhielt eine Zeit lang im Kochen. Es konnte jedoch hier nicht wie bei der Bestimmung des spec. Gewichts verfahren werden; denn wenn sich auch in $1 / 4^{--1 / 2}$ Stunde der Niederschlag zu Boden setzte, so ist es ja bekannt, dass durch blosses Weggiessen der über ihm stehenden Lösung viele Partikelchen desselben mit fortgerissen werden, wodurch eine ganz unzulässige Ungenauigkeit entstände.

Wollte man jedoch hierbei so behutsam verfahren, dass dieser Uebelstand nicht eintritt, so müsste noch soviel Lösung neben dem Niederschlag im Becherglase bleiben, dass 2 Pyknometer-Füllungen kaum hingereicht hätten, um diese Menge Flüssigkeit aufzunehmen, wodurch natürlich erstens die Arbeit bedeutend verlängert und zweitens die Ungenauigkeit verdoppelt worden wäre.

Ich brachte deshalb den Niederschlag auf ein Filter und liess das Filtrat in meine untergestellte Spritzflasche laufen, die ich erst zweimal mit demselben ausspülte, um die an den Wänden hängende Feuchtigkeit zu verdrängen. Den auf dem Filter befindlichen Niederschlag, welcher natürlich mit derselben Lösung, die sich in der Spritzflasche befand, durchtränkt war, versuchte ich nun mit Hülfe der letzteren, natürlich unter Anwendung eines Trichters, ins Pyknometer zu spülen. Es traten jedoch hierbei unangenehme Uebelstände auf. Denn da beim blossen Spritzen mit der Lösung viel mehr von derselben verbraucht worden wäre, als das Pyknometer gefasst hätte, so sah ich mich genöthigt mit einem dünnen Glasstabe zu Hülfe zu kommen, der auch dazu benutzt werden 
musste, die vom Filter gelösten Stïcke durch die Trichteröffnung zu bringen, bei welcher unangenehmen Operation sich aber viel Luftbläschen bildeten.

Da die auf diese Weise abgelöste Thonerdemasse sich nicht genügend in der übrigen Flüssigkeit suspendirte, sondern zum grossen Theil als gesonderte Klumpen im Pyknometer herumschwamm, so stiegen natürlich die von diesen Klumpen mechanisch eingeschlossenen Luftbläschen nicht zum Hals des Pyknometexs empor und konnten also auch nicht entfernt werden.

Fig. 5.

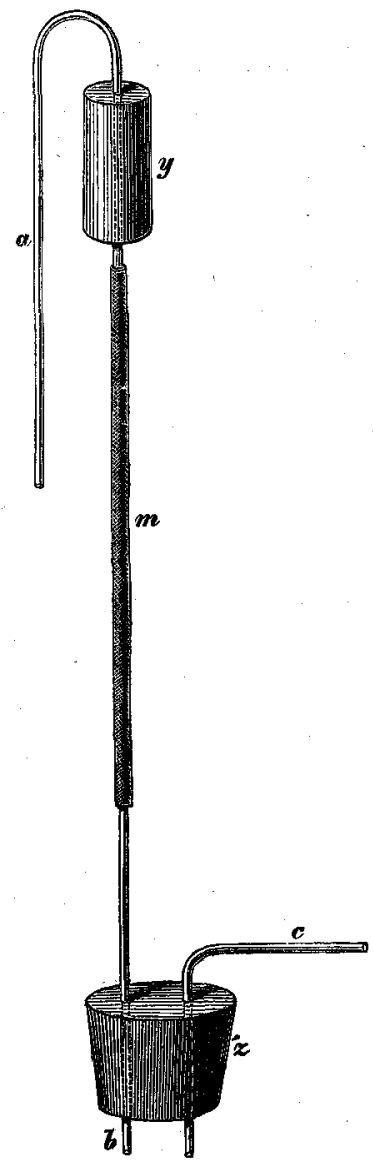

Die bis hierher beschriebene Methode musste ich also fallen lassen, und es ist mir seitdem wirklich glücklich gelungen, mit Hülfe einer Saughebervorrichtung die über dem Niederschlage stehende Flüssigkeitssäule fast bis auf den letzten Tropfen von demselben zu entfernen, ohne dass deshalb eine Spur des Niederschlags mit übergegangen wäre. Einen gewöhnlichen Saugheber anzuwenden würde aber hierbei unangenehm gewesen sein, denn das Füllen desselben hätte nicht mit Wasser, sondern mit derselben Flüssigkeit, welche abgezogen werden sollte, geschehen müssen. Ich construirte mir deshalb einen Heber, wie nebenstehende Figur zeigt, in welcher $a, b$ und c Glasröhren, $m$ einen Gummischlauch and $y$ und $z$ Korke darstellen, von denen $z$ auf meine gewöhnliche Spritzflasche passt, und wie Figur 5 zeigt, zweifach durchbohrt ist.

Ich stellte nun das Becherglas, aus welchem ich mit Hülfe dieser Vorrichtung die über dem Niederschlage befindliche Flüssigkeit abziehen wollte, nachdem sich derselbe vollständig zu Boden gesetzt hatte, auf ein Stativ und befestigte an einem ebenfalls an diesem Stativ befindlichen Halter (der in Figur 6 der Uebersichtlichkeit wegen 
weggelassen ist) den Kork y, so dass der eine Schenkel der Glasröhre a in die Flüssigkeit des Becherglases hineinragte und mit seinem unteren

Fig. 6.

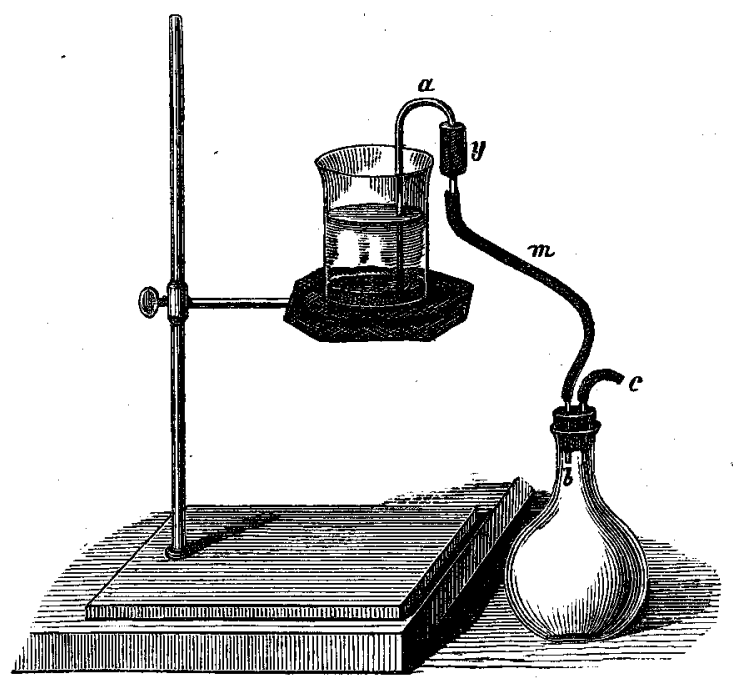

Ende vielleicht $2 \mathrm{Cm}$. über dem Niveau des Niederschlags stand, wie ich in Fig. 6 darzustellen versucht habe.

Den Kork $\mathrm{z}$ befestigte ich an meiner Spritzflasche und sog einen Augenblick mit dem Munde an der Röhre c. Sofort begann die Flüssigkeit continuirlich durch die Hebervorrichtung in die Spritzflasche hinabzulaufen. Das zuerst Durchlaufende be-

nutzte ich, um sie zweimal damit auszuspülen.

Das Stativ war von vorn herein für alle Versuche so eingestellt worden, dass die obere Grenze des Niederschlags sich nicht mehr als $1-2 \mathrm{Cm}$. über dem unteren Ende der an der Spritzflasche befindlichen Röhre b befand und war ausserdem das Becherglas etwas schief gestellt, weshalb sich das Niveau des Niederschlags an der Stelle, wo die Röhre a eintauchte, am tiefsten befand.

Die Lösung lief nun in wenigen Minuten aus dem Becherglase in den Kolben; jedoch wurde, sobald sich das Flüssigkeitsniveau dem unteren Ende von $\mathbf{b}$ näherte, der Lauf der Flüssigkeit, wegen der oben angeführten Einstellung des Stativs, sehr langsam, weshalb ich die Röhre a jetzt unbedenklich so weit herabschieben konnte, dass ihr unteres Ende nur wenige Millimeter vom Niederschlage entfernt war, ohne dass, bei dem jetzt so schwach gewordenen Zuge des Hebers, eine einzige Flocke mit übergegangen wäre.

Da nun ausserdem wegen der schrägen Stellung des Becherglases der Niederschlag sich an der Stelle, wo der Heber eintauchte, am tiefsten befand, so war es auf diese Weise wirklich möglich, die über ihm stehende klare Lösung fast bis auf den letzten Tropfen von demselben zu trennen. 
Ich entfernte nun die Hebervorrichtung und setzte auf die, nun mit der Lösung angefüllte, Spritzflasche wieder den gewöhnlichen Gummistopfen mit Spritzvorrichtung.

Mit dieser Spritzflasche spülte ich nun das Pyknometer einige Male aus, um die darin enthaltene Feuchtigkeit vollständig zu verdrängen, füllte es hierauf mit der in der Spritzflasche enthaltenen Flüssigkeit an, bestimmte die Temperatur derselben und wog es. (Ein vorher angestellter Versuch hatte dargethan, dass die Lösung beim Erhöhen der Temperatur sich fast ebenso ausdehne wie Wasser, wenigstens innerhalb so kleiner Temperaturgrenzen, wie sie hier vorkommen konnten.) Hierauf brachte ich wieder in ähnlicher Weise wie schon früher angeführt, einen Trichter mit möglichst enger Ausflussröhre über das Pyknometer und goss den im Becherglase zurückgebliebenen Niederschlag durch denselben. Ich spülte das Becherglas hierauf mit der in meiner Spritzflasche befindlichen Lösung mehrmals aus, um sämmtlichen an den Wänden hängenden Niederschlag ins Pyknometer zu bringen, welches ich nun mit der Flüssigkeit anfüllte, und einige Minuten wartete, bis sich darin der etwas aufgerührte Niederschlag wieder ein wenig gesetzt hatte. Hierauf bestimmte ich auch hier die Temperatur der Flüssigkeit und wog nun das Pyknometer.

Abgesehen davon, dass auf diese Weise derartige unzulässige Fehlerquellen, wie bei dem vorigen Verfahren vollständig vermieden wurden, dauerte auch diese ganze Operation nicht halb so lange; denn das auf das Filter Bringen der Thonerde, sowie das Herabspülen derselben ins Pyknometer nahm im Vergleich zur Operation mit dem Sangheber sehr viel Zeit in Anspruch, wobei ausserdem die Operation viel unangenehmer war.

Der bis hierher beschriebene Versuch wurde mit vier Proben zu je 50 CC. vorgenommen, wobei sich folgende Zahlen ergaben:

$$
\text { I. Analyse. }
$$

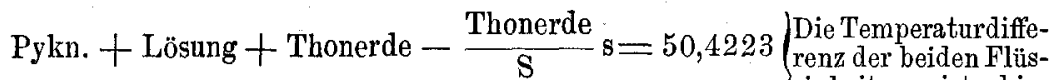

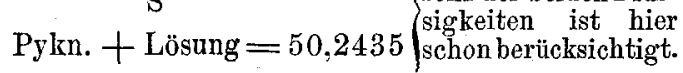
Thonerde $-\frac{\text { Thonerde }}{\mathrm{S}} \mathrm{s}=0,1788$.

Die hier gefundene Zahl 0,1788 entsprach also in Formel

dem Ausdruck $\mathrm{G}-\mathrm{g}$.

$$
\text { Al } \theta_{3} H_{3}=\frac{S}{S-s}(G-g)
$$


Der Werth s würde sein

$$
\frac{\mathrm{g}-\mathrm{P}}{\mathrm{V}}
$$

wenn P die Tara und V das Volumen des Pyknometers bezeichnet, folglich

Es wäre also

$$
\mathrm{s}=\frac{50,2435-9,914}{40,170}=1,0038 \text {. }
$$

$$
\mathrm{Al} \theta_{3} \mathrm{H}_{3}=\frac{2,214}{2,214-1,0038}=0,1788
$$

oder, da wir ja die Thonerde als Anhydrid berechnen müssen, um sie mit der später vorzunehmenden Gewichtsanalyse vergleichen zu können,

$$
\mathrm{Al}_{2} \theta_{3}=\frac{2,214 \cdot 0,1788}{1,2102} \cdot \frac{51,4}{78,4} .
$$

In diesem Ausdruck wären allerdings nicht weniger als 3 Multiplikationen and eine Division vorzunehmen. Jedoch würde er sich auf logarithmischem Wege, besonders da das spec. Gewicht 2,214 und die Molekulargewichte 51,4 und 78,4 für jeden Fall dieselben bleiben, in wenigen Minuten ganz bequem berechnen lassen.

Es ergab sich auf diese Weise

$$
\mathrm{Al}_{2} \theta_{3}=0,2145
$$

oder, wenn man berücksichtigt, dass die angewandten 50 CC. 2 Gramm Alaun entsprechen

$$
\begin{aligned}
& \mathrm{Al}_{2} \theta_{3}=10,73 \% . \\
& \text { II. Analys e. }
\end{aligned}
$$

$\mathrm{P}+\mathrm{L}+\mathrm{Th}-\frac{\mathrm{Th}}{\mathrm{S}} \mathrm{s}=50,4737$ Die Temperaturdifferenz ist ebenfalls $\mathrm{P}+\mathrm{L}=50,2960 \quad$ schon berücksichtigt.

$$
\mathrm{Th}-\frac{\mathrm{Th}}{\mathrm{S}} \mathrm{s}=0,1777 \text {. }
$$

Der Werth s wäre hier $=$

$$
\frac{50,296-9,914}{40,17}=1,0053
$$

Man bekäme also für $\mathrm{Al}_{2} \theta_{3}$

Dies gibt berechnet:

$$
\mathrm{Al}_{2} \theta_{3}=\frac{2,214 \cdot 0,1777}{1,2087} \cdot \frac{51,4}{78,4} \text {. }
$$

$$
\begin{aligned}
& \mathrm{Al}_{2} \Theta_{3}=0,2135 \\
& \mathrm{Al}_{2} \theta_{3}=10,67 \% .
\end{aligned}
$$


ohne Auswaschen und Trocknen derselben.

III. Analyse.

$$
\begin{aligned}
\mathrm{P}+\mathrm{L}+\mathrm{Th}-\frac{\mathrm{Th}}{\mathrm{S}} \mathrm{s} & =50,6585 \\
\mathrm{P}+\mathrm{L} & =50,4810 \\
\mathrm{Th}-\frac{\mathrm{Th}}{\mathrm{S}} \mathrm{s} & =0,1775 .
\end{aligned}
$$

Hier würde sich für $s$ ergeben

$$
\begin{gathered}
\frac{50,481-9,914}{40,17}=1,010 . \\
\mathrm{Al}_{2} \theta_{3}=\frac{2,214 \cdot 0,1775}{1,204} \cdot \frac{51,4}{78,4} \\
\mathrm{Al}_{2} \theta_{3}=0,2140 \\
\mathrm{Al}_{2} \theta_{3}=10,70 \% . \\
\text { IV. A naly se. } \\
\mathrm{P}+\mathrm{L}+\mathrm{Th}-\frac{\mathrm{Th}}{\mathrm{S}} \mathrm{s}=50,5975 \\
\mathrm{P}+\mathrm{L}=50,4205 \\
\mathrm{Th}-\frac{\mathrm{Th}}{\mathrm{S}} \mathrm{s}=0,1770 \\
\mathrm{~s}=\frac{50,4205-9,914}{40,17}=1,008 \\
\mathrm{Al}_{2} \theta_{3}=\frac{2,214.0,177}{1,206} \cdot \frac{51,4}{78,4} \\
\mathrm{Al}_{2} \theta_{3}=0,2118 \mathrm{Grm} . \\
\mathrm{Al}_{2} \theta_{3}=10,59 \% .
\end{gathered}
$$

Die hier gefundenen vier Resultate stimmen wirklich gar nicht schlecht überein, und man sieht hieraus, dass wenigstens für technische Analysen das Verfahren vollständig genügend ist.

Um ganz sicher über die Richtigkeit der Resultate zu sein, bestimmte ich bei zwei dieser Analysen den im Pyknometer befindlichen Niederschlag noch auf dem gewöhnlichen Wege und fand hier

$$
0,215 \text { und } 0,214 \text { Grm. }
$$

oder

$$
\mathrm{Al}_{2} \theta_{3}=10,75 \text { und } 10,70 \% \text {, }
$$

was einen Durchschnittswerth von

ergibt.

$$
10,72 \%
$$


Dass dieser Werth etwas geringer ausfiel, als er nach der Formel des Alauns gefunden werden musste $(10,83 \%)$, rührt jedenfalls von etwas Feuchtigkeit her, welche den Krystallen noch anhaftete.

Vergleichen wir nun die vier Werthe $10,73,10,67,10,70$ und 10,59 mit dem gewichtsanalytisch gefundenen Mittelwerth 10,72, so sehen wir, dass auch hier keine zu bedeutenden Abweichungen stattfinden.

\section{Genauigkeitsregeln. *)}

Da man die Spritzflasche, an welcher die Hebervorrichtung angebracht ist, erst mehrmals mit 'der Lösung ausspülen muss, so ist es natürlich nöthig, dass sich während dessen ein kleines, Becherglas daneben befindet, um mit diesem, während des Ausspülens, den continuirlich laufenden Flüssigkeitsstrahl auffangen zu können.

Da die Temperatur der Lösung eine grosse Rolle spielt (es betrug z. B. wie schon erwähnt für mein Pyknometer bei Erhöhung um 10, die Gewichtsabnahme 8 Milligramm), so muss sie natürlich bis auf $0,1^{0}$ abgelesen werden. Um dies mit Genauigkeit thun zu können, ist es nöthig, dass die Thermometerkugel 1-2 Minuten im Pyknometer bleibt. Am besten ist es, während dieser Zeit das Thermometer an irgend ein Stativ zu lehnen.

Bei all' diesen Operationen darf man, der Körperwärme wegen', das Pyknometer (falls dasselbe nicht 2 Röhrenansätze haben sollte) so wenig wie möglich mit der Hand anfassen und ist es deshalb am besten, dasselbe in eine Porzellanschale zu setzen und, wenn nöthig, diese statt des Pyknometers zu ergreifen.

Da die Lösung im Becherglase nach dem Absitzen des Niederschlags meistens noch warm ist, so muss dasselbe einige Minuten in kaltem - aber nicht zu kaltem - Wasser abgekühlt werden; denn die Wärme der Lösung darf um nicht mebr als $2^{0}$ von der Luftwärme differiren, indem sonst, noch bevor man die Temperatur im Pyknometer abliest, dasselbe sich an den Wänden abkühlen würde, während in der Mitte, wo sich die Thermometerkugel befindet, die Wärme noch etwas höher bliebe. Die Folge davon würde das Ablesen einer zu hohen Temperatur sein. Es wäre deshalb auch nicht unzweckmässig, wenn im Laboratorium ein besonderes Thermometer die Temperatur der Luft anzeigte.

*) Die gewöhnlichen schon längst bei Anwendung von Pyknometern gebräuchlichen Sicherheitsmaassregèln, wie z. B. das Abkühlen derselben nach dem Füllen, müssen hier als bekannt vorausgesetzt werden. 
Aus dem vorhin angeführten Grunde darf man auch das Pyknometer, sobald sich der Niederschlag darin nur einigermassen zu Boden gesetzt hat, so dass wenigstens der oberste Theil dieses kleinen Gefässes von demselben frei geworden ist, nicht länger stehen lassen, sondern muss, nachdem man die Temperatur gemessen, es jetzt füllen und wägen. Wenn auch hierbei die über dem Niederschlag im Pyknometer befindliche Flüssigkeit noch getrubt ist und deshalb beim Aufsetzen des Glasstopfens (welcher nur sanft aufgedrückt werden darf) die ausfliessenden Tropfen noch Spuren von dem ersteren enthalten, so hat dies auf die Genauigkeit so gut wie gar keinen Einfluss.

Den nach dem Aufsetzen des Stopfens auf dem Röhrenansatze befindlichen Tropfen entfernt man am besten mit dem Finger (nicht durch Sangen mit Fliesspapier).

Eine fernere Fehlerquelle würde entstehen, wenn man das Pyknometer mit dem darin Befindlichen in die Nähe einer Gasflamme setzen wollte, indem dieselbe ebenfalls die Temperaturerhöhung eines Theils der Flüssigkeit bewirken würde.

Es muss deshalb, falls man am Abend arbeitet, darauf gesehen werden, dass das Messgefäss wenigstens 2 bis 3 Meter von den vorhandenen Flammen entfernt ist.

Fig. 7.

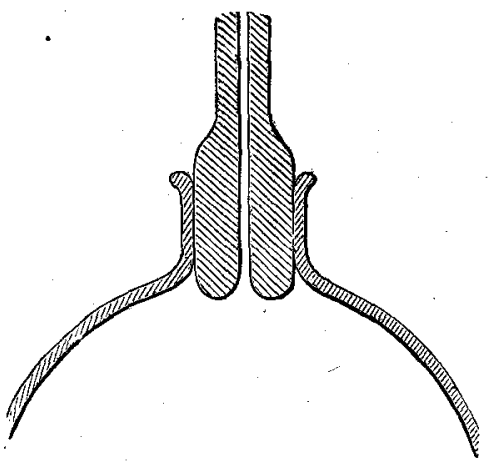

Noch wäre zu erwähnen, dass viel auf ein genaues und nicht zu kleines Pyknometer ankommt. (Dasselbe darf nicht unter $30 \mathrm{CC}$. Rauminhalt besitzen). Dr. Geissler in Bonn a. Rh. versteht diese Instrumente sehr genau anzufertigen.

Ein Pyknometer, welches einen Hals mit gekrümmtem Rande besitzt, wie ihn Figur 7 im Durchschnitt zeigt, bewährt sich nicht gut, indem sich dasselbe an diesem Rande schlecht abtrocknen lässt. Man muss deshalb hier den Hals etwas abschleifen, so dass derselbe die in Fig. 8 dargestellte Form annimmt. Da sich ferner das Trocknen eines Pyknometers mit Fliesspapier unbequem bewerkstelligen lässt, so ist es am besten, hier 2 Tücher an- 
zuwenden. Man trocknet es erst mit dem einen so gut als möglich und entfernt mit dem andern kurz vor der Wägung die letzten Reste von

Fig. 8.

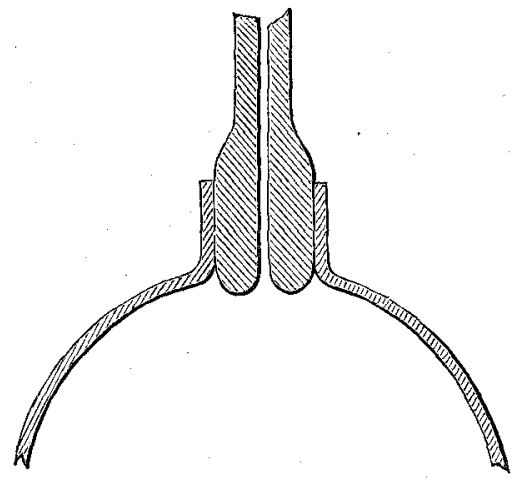

Feuchtigkeit, Natürlich darf das zweite dieser beiden Tucher, welches immer eine gewisse Trockenheit besitzen muss, nie die Function des ersten übernehmen. Zwei Regeln, welche vielleicht nur bei der Bestimmung der Thonerde gelten, sind ausserdem, dass ein $\mathrm{Ab}$ kühlen der Lösung vor dem vollständigen Absitzen des Niederschlags hier nicht zulässig ist und dass die Flüssigkeitssäule nicht

länger als 2-4 Stunden über demselben stehen bleiben darf, indem er eine (wenn auch geringe) Adhäsion auf die, in der Lösung befindlichen Salze ausübt, wodurch das spec. Gewicht der in der Nähe des Niederschlags befindlichen Flüssigkeit ein wenig höher wird, als das der darüberstehenden.

Aus diesem Grunde ist es auch nicht gerathen, die Fällung in einer sehr salzreichen Lösung vorzunehmen, ohne dieselbe vorher genügend zu verdünnen.

Hütet man sich vor allen hier angeführten Fehlerquellen, (was ja gar keine Schwierigkeiten hat, indem die Operationen ebenso rasch, ja fast noch schneller vor sich gehen, als wenn man diese Vorsichtsmaassregeln ausser Acht lässt) so werden die Resultate immer günstig ausfallen.

Natürlich würden sich fast alle vorkommenden Niederschläge in derselben Weise, wie hier die Thonerde, quantitativ bestimmen lassen, womit selbstverständlich durchaus nicht gemeint ist, dass dieșes Verfahren überall den anderen vorzuziehen sei.

Gibt es doch für die Bestimmungen vieler Substanzen so zweckmässige maassanalytische Methoden, dass diese wohl ohne Zweifel den Vorzug verdienen, So würde z. B. das Fällen einer Kalklösung mit oxalsaurem Ammon und Bestimmen des überschüssig zugesetzten Fällungsmittels in einem aliquoten Theile des Filtrats, wobei man ja den Niederschlag auch nicht auszuwaschen braucht, wohl noch rationeller sein, als obiges Verfahren. 
Ungeachtet dessen dürfte dasselbe jedoch in manchen Fällen eine angenehme Erleichterung bieten, indem bekanntlich für viele Körper die maassanalytischen Methoden (besonders wenn es nöthig ist diese Körper erst von anderen, die irgend wie störend einwirken, zu trennen) ebenfalls sehr mühevoll sind.

Uebrigens gedenke ich die oben beschriebenen Versuche auch auf andere Körper auszudehnen und die gefundenen Resultate der Oeffentlichkeit zu übergeben.

Als Schluss möchte ich jetzt nur noch Folgendes anführen:

Will man in einer Substanz mehrere Bestandtheile quantitativ ermitteln, sodass es nöthig ist, dieselben von einander zu trennen, so kann anch hier das Auswaschen vollständig umgangen werden. Angenommen, es sei in einer Lösung Kupfer und Blei enthalten, welche Körper sich natürlich am zweckmässigsten durch Versetzen mit Schwefelsäure von einander scheiden lassen würden; so kann man ja nach dem Bestimmen des schwefelsauren Bleies mit Hülfe eines Pyknometers von der noch übrig bleibenden Lösung einen aliquoten Theil (vielleicht $9 / 10$ ) zur Kupferbestimmung verwenden und das gefundene Resultat mit der diesem aliquoten Theile entsprechenden reciproken Zahl (also hier 10/9) multipliciren, welches Verfahren ja auch bei der Maassanalyse in manchen Fällen angewendet wird. Der kleine Fehler, welcher hierbei dadurch begangen wird, dass man das Volumen des Bleiniederschlags ausser Acht lässt, kommt offenbar nicht in Betracht, könnte übrigens mit Leichtigkeit vermieden werden, indem man ja dieses Volumen aus dem gefundenen Gewichte und dem als bekannt vorausgesetzten spec. Gewichte durch einfache Division finden kann.

Natürlich lässt sich eine derartige Trennungsmethode für alle aus einer Lösung fällbaren Körper anwenden, gleichviel in welcher Anzahl sie in dieser Lösung vorhanden sind.

Selbstverständlich kann alles bisher Angeführte ausser zur Ermittlung des Gewichtes irgend welcher Niederschläge auch zur Bestimmung von Rückständen verwendet werden. So wtirde z. B. in einer mit Schwerspath gemengten Farbe, wie Mennige, chromsaures Blei, Zinkweiss und dergl. mehr durch Behandeln derselben mit Salzsäure und Bestimmen des Rückstandes im Pyknometer, sich leicht dessen Monge ermitteln und der Werth der Farbe danach bestimmen lassen.

Dresden, im December 1876. 